\title{
Models for solar magnetic loops
}

\section{Comparison with SOHO-CDS observations on the solar disk}

\author{
A. Brković ${ }^{1}$, E. Landi ${ }^{2,3}$, M. Landini ${ }^{4}$, I. Rüedi ${ }^{5}$, and S. K. Solanki ${ }^{2}$ \\ 1 Institute of Astronomy, ETH-Zentrum, Zürich, Switzerland \\ 2 Max-Planck-Institute für Aeronomie, Katlenburg-Lindau, Germany \\ 3 Naval Research Laboratory, Washington DC, 20375-5320, USA \\ 4 Dipartimento di Astronomia e Scienza dello Spazio, Università di Firenze, Italy \\ ${ }^{5}$ Physikalisch-Meteorologisches Observatorium Davos, World Radiation Center, 7260 Davos Dorf, Switzerland
}

Received 27 October 2000 / Accepted 28 November 2001

\begin{abstract}
The present work describes a detailed comparison between SOHO-CDS observations of active region loops with a static, isobaric loop model developed assuming a temperature-independent heating function in the energy balance equation and a variable loop cross-section. The loop model is described in Landini \& Landi (2002). Observations of an active region recorded by CDS have been analyzed. Additional data from the EIT and MDI instruments on board the SOHO satellite, and broad band soft X-rays images from the Yohkoh satellite, have been used to complement the CDS dataset. CDS monochromatic images from lines at different temperatures have been co-aligned with EIT, MDI and Yohkoh images and a loop structure has been identified. Two other loop structures are visible but their footpoints are not clearly identified, and have not been analyzed. Electron density, temperature and pressure along the selected loop structure have been measured by means of line ratio techniques. These quantities have been used to test the assumption of constant pressure adopted in the theoretical model, and to compare their values with its predictions. The loop filling factor has also been estimated from the CDS data after assumptions on the loop geometry have been made. Comparison with CDS data has shown that a classical model is not able to reproduce the observations; despite the large uncertainties, mainly given by the limited CDS spatial resolution, indications suggest that agreement occurs only if an "ad hoc" isothermal region is added on top of the loop and a large conductive flux at the base is assumed. Suggestions for improvements of theoretical loop models and further studies with the EIS instrument on Solar-B, due for launch in 2005, are given.
\end{abstract}

Key words. Sun: corona - techniques: miscellaneous

\section{Introduction}

In the last twenty years a number of space missions, such as Skylab, the Solar Maximum Mission (Solar Physics 1980) and Yohkoh (Ogawara et al. 1991) have been devoted to the study of the solar corona, with the aim of understanding its structure and discovering the processes that heat its plasma. However, most of these missions were equipped with broad band imaging instruments with increasingly higher spatial resolution, but rather poor spectral resolution. Even more recent space projects such as the SOHO and TRACE missions carried several broadband imaging instruments devoted to the investigation of

Send offprint requests to: E. Landi, e-mail: landi@poppeo.nrl.navy.mil the chromosphere, transition region and corona with high spatial detail, but very limited spectral resolution.

These modern imaging instruments have been used to study plasma loops. Temperature diagnostics using filter ratios from the SOHO-EIT (Delaboudinière et al. 1995) and Yohkoh-SXT (Tsuneta et al. 1991) have allowed several authors (Neupert et al. 1998; Aschwanden et al. 1999, 2000a; Lenz et al. 1999; Priest et al. 1998, 2000) to investigate the plasma temperature profile along loops. EITderived temperatures for coronal loops have been found to be nearly constant for most of the loop lengths, indicating small conductive fluxes, so that the energy lost by conduction is much smaller than the energy lost by radiation.

The possibility of spatially resolving many loops has also allowed the well-known length-vs.-temperature 
scaling law to be investigated: the results show that the relation found by Rosner et al. 1978 (RTV) is not always supported by the observations (Aschwanden et al. 1999, 2000a; Porter \& Klimchuk 1995; Klimchuk \& Porter 1995; Kano \& Tsuneta 1995, 1996; Kankelborg et al. 1996, 1997). These results have led Aschwanden et al. (1999, 2000a) to argue that coronal loops with temperatures in the range $1-2.5 \times 10^{6} \mathrm{~K}$ are not under steady-state conditions and that the RTV-like models should be revised. However, Reale \& Peres (2000) suggested that the very low temperature gradient could be due to the presence of filamentation, and can be simulated by the presence of several unresolved RTV-like loops at different temperatures: this view is consistent with the results from TRACE, showing that loops are composed of many individual filaments, down to the telescope spatial resolution limit (Handy et al. 1999). Oluseyi et al. (1999) developed quasi-static loop models using different boundary conditions at the base of the transition region from RTV and found three classes of solutions, one of which shows that the loop is radiationdominated and the temperature gradient along its length is small.

Priest et al. $(1998,2000)$ investigated the shape of the unknown heating function by relating the temperature profile along the loop length with the spatial distribution of the unknown heating function. They compare their predictions with temperature profiles obtained on large coronal loops seen by Yohkoh at the solar limb using filter ratio techniques. They find that the observations are best reproduced by a uniform heating function. On the other hand, Aschwanden et al. (2000b) found that multi-thread models of loops in the temperature range of $T_{\mathrm{e}} \simeq 0.8-1.6 \times 10^{6} \mathrm{~K}$ are consistent with observations only if the heating function is non-uniform, with a scale height of $(17 \pm 6) \times 10^{3} \mathrm{~km}$. Subsequent work has confirmed this result, and revised the heating function scale-height value to $(12 \pm 5) \times 10^{3} \mathrm{~km}$. In general, loops observed by Yohkoh are hotter than those seen by EIT, and they often show a significant energy conduction which can be of the same size of, or greater than radiative losses (Kano \& Tsuneta 1995, 1996; Porter \& Klimchuk 1995; Klimchuk \& Porter 1995).

Detailed measurements of plasma properties along loop lengths using monochromatic images and line ratio techniques are surprisingly scarce, despite the great opportunities given by the two SOHO spectrographs CDS (Harrison et al. 1995) and SUMER (Wilhelm et al. 1995). Most of the attention has been focused on velocity studies in active region loops, and several authors have found signatures of highly dynamic behaviour and variability of transition region and coronal loops (Brekke et al. 1997a; Fludra et al. 1997; Kjeldseth-Moe \& Brekke 1998; Spadaro et al. 2000; Chae et al. 2000). Little attention has been paid to density, temperature and abundance measurements using line ratios, despite the fact that this technique allows more precise measurements than ratios between broad band filters like those on EIT, Yohkoh and TRACE. To our knowledge, the only attempt has been made by Landini et al. (1999) using CDS observation of an active region loop system on the disk.

Theoretical loop models have been long investigated by a variety of authors, leading to a large body of work; a brief summary can be found in Landini \& Landi (2002, hereafter Paper I). Despite all the efforts, however, loop models still are not able to explain the observations, the processes that heat the plasma inside loops have not been fully understood, and no conclusion has been reached on the shape of the heating function.

The aim of the present work is to carry out a thorough comparison of the loop model developed in Paper I with observations of an active region loop system, obtained with the CDS instrument on board SOHO. Additional data from the EIT and MDI instruments on board SOHO and Yohkoh-SXT are used to provide context images and to help in the selection of loop structures. First, density and temperature measurements are obtained from line ratios from the CDS instrument; the study of the emission measure (EM) along the loop structure allows a rough estimate of the loop structure filling factor. Then, a comparison is carried out using the simple diagnostic technique developed in Paper I, that allows the determination of the main physical quantities of the observed loops, using spectral line intensities from CDS. In the light of the results, we also discuss some improvements required by the model in order to better reproduce the observations. The uncertainties in the loop shape selection and their effects on the results are also discussed, and future studies are proposed to be carried out with future instruments having higher spatial and temporal resolution.

The present paper is structured as follows: Sect. 2 illustrates the observations; Sect. 3 describes the loop selection and the plasma diagnostic results obtained by means of line ratio techniques. A comparison between the model described in Paper I and observations is carried out in Sect. 4 ; in this section we also discuss the presence of an isothermal region on top of the loop model and the initial conditions for the conductive flux at the loop base. In Sect. 5 the results are discussed and new studies are proposed. Section 6 summarizes the conclusions.

\section{The observations}

The observations were recorded on April 29th 1997 with the Coronal Diagnostic Spectrometer (CDS, Harrison et al. 1995), the Extreme-ultraviolet Imaging Telescope (EIT, Delaboudinière et al. 1995), the Michelson Doppler Interferometer (MDI, Scherrer et al. 1995) and the Soft X-ray Telescope (SXT, Tsuneta et al. 1991) on Yohkoh, although not exactly co-temporally. The CDS observations have been carried out using the $2^{\prime \prime} \times 240^{\prime \prime}$ slit on the Normal Incidence Spectrometer (NIS). Between 8:20 and 10:40 UT the CDS slit scanned an active region on the solar disc centred at around $\left(-100^{\prime \prime},-400^{\prime \prime}\right)$ in the solar disc coordinate system. This active region has the advantage of showing prominent but at the same time also simple loop configurations. A total of 120 exposures 

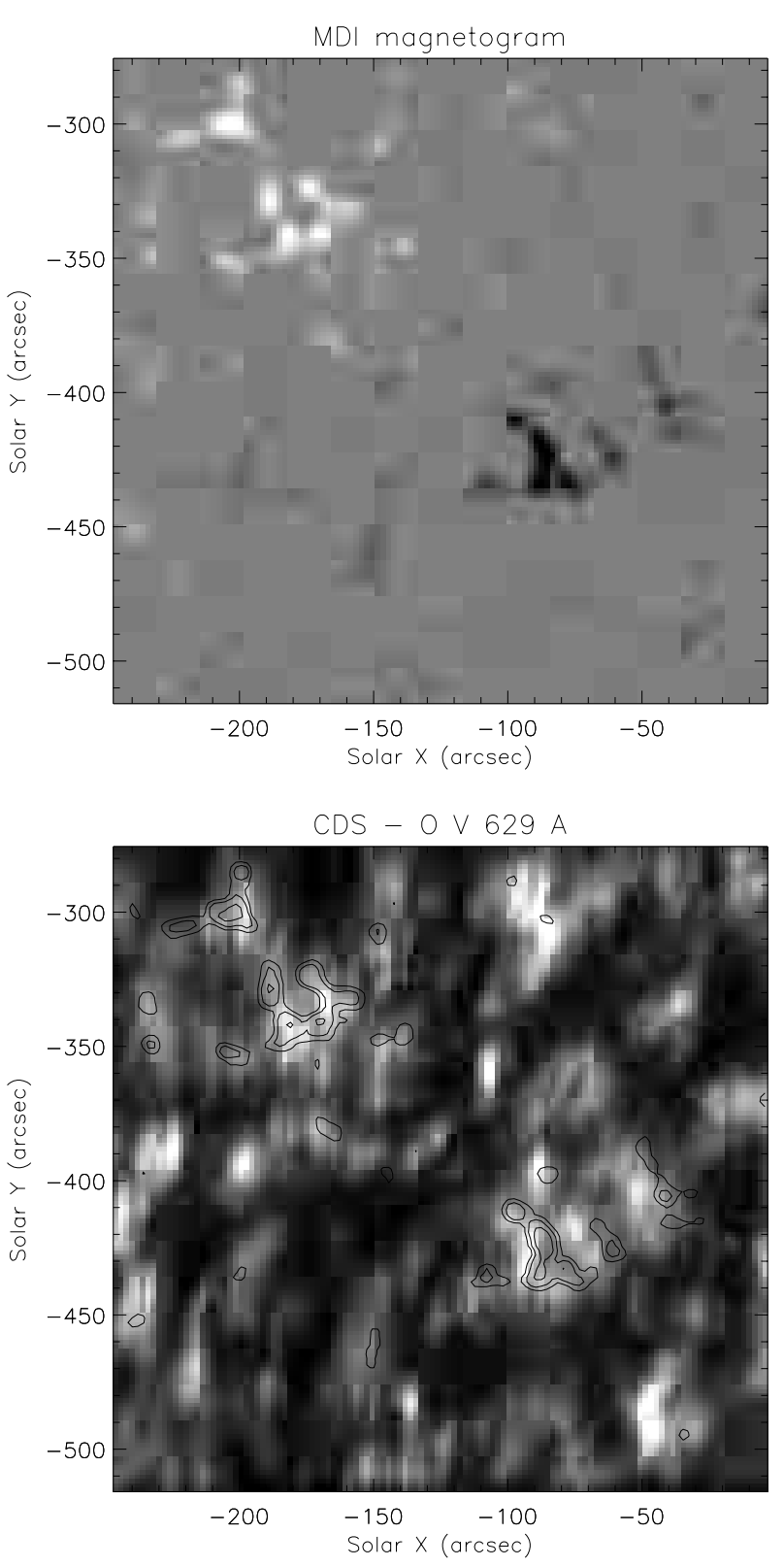

Fig. 1. Top: the magnetic field map of the region measured by MDI on SOHO; white is positive polarity, black is negative polarity. Bottom: intensity map obtained with CDS in the O v $629.73 \AA$ line, formed at around $3 \times 10^{5} \mathrm{~K}$. Magnetic field contours from MDI are superimposed on the OV map.

were recorded with $2.03^{\prime \prime}$ steps along the solar E-W direction, so that the size of the resulting field of view was $243.6^{\prime \prime} \times 240.2^{\prime \prime}(120 \times 143$ pixels $)$, with the pixel size being $2.03^{\prime \prime} \times 1.68^{\prime \prime}$. The exposure time for each slit position was $60 \mathrm{~s}$.

In order to maintain the high cadence of the observations 16 spectral windows were extracted, containing a total of 19 spectral lines of interest for our analysis. These lines are listed in Table 1, together with estimates of their formation temperatures. The observed lines come from the Chromosphere (HeI), the Transition Region (O III, Ov, Ne v, Mg VII) and the Corona (Mg VIII, Mg IX, Si XI, Si XII, FexII, FexiII, FexIV and Fexvi). The selected sample
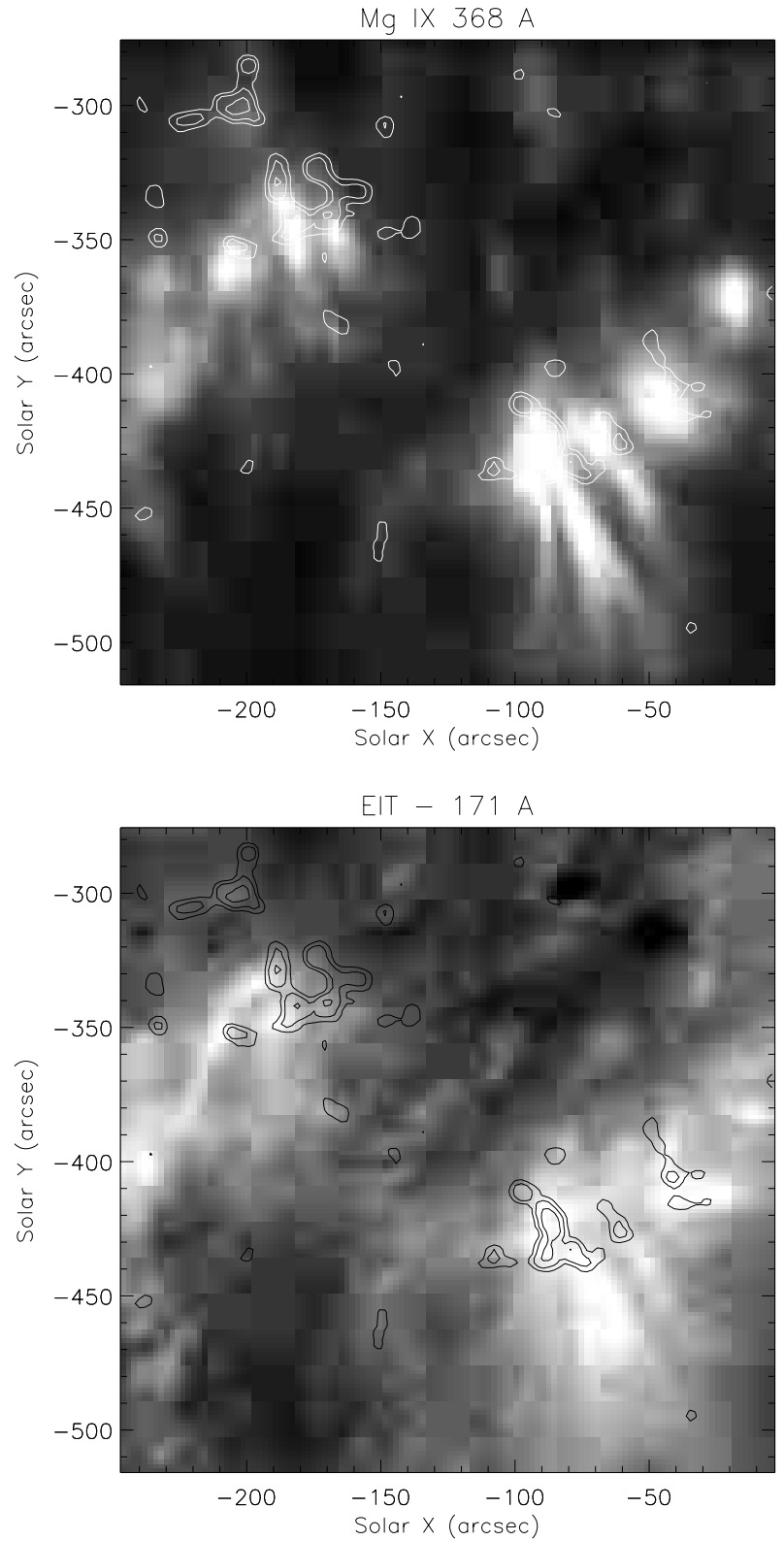

Fig. 2. Top: intensity map obtained with CDS in the Mg IX $368.07 \AA$ line, formed at around $10^{6} \mathrm{~K}$. Bottom: intensity map obtained with the EIT $171 \AA$ channel. In active region the emission is mostly due to plasma at around $1.3 \times 10^{6} \mathrm{~K}$. Magnetic field contours from MDI are superimposed.

allows the electron density to be measured using pairs of Fe XII and Fe XIII lines. The presence of consecutive stages of ionization for several elements allows temperature diagnostics through intensity ratios of lines of different ions.

The EIT observations provide us with full disc filtergrams in 4 spectral lines, the He II line at $304 \AA\left(8 \times 10^{4} \mathrm{~K}\right)$, the Fe IX-X line at $171 \AA\left(1.3 \times 10^{6} \mathrm{~K}\right)$, the Fe XII line at $195 \AA\left(1.6 \times 10^{6} \mathrm{~K}\right)$ and the $\mathrm{Fe} \mathrm{Xv}$ line at $284 \AA\left(2 \times 10^{6} \mathrm{~K}\right)$. The filtergrams were taken consecutively between 07:00 and 07:20 UT. The pixel size was $2.59^{\prime \prime} \times 2.59^{\prime \prime}$. SXT also provides full disc filtergrams, but is sensitive to higher temperatures than EIT. The pixel size of SXT images was $4.91^{\prime \prime} \times 4.91^{\prime \prime}$ and observations took place between 08:00 

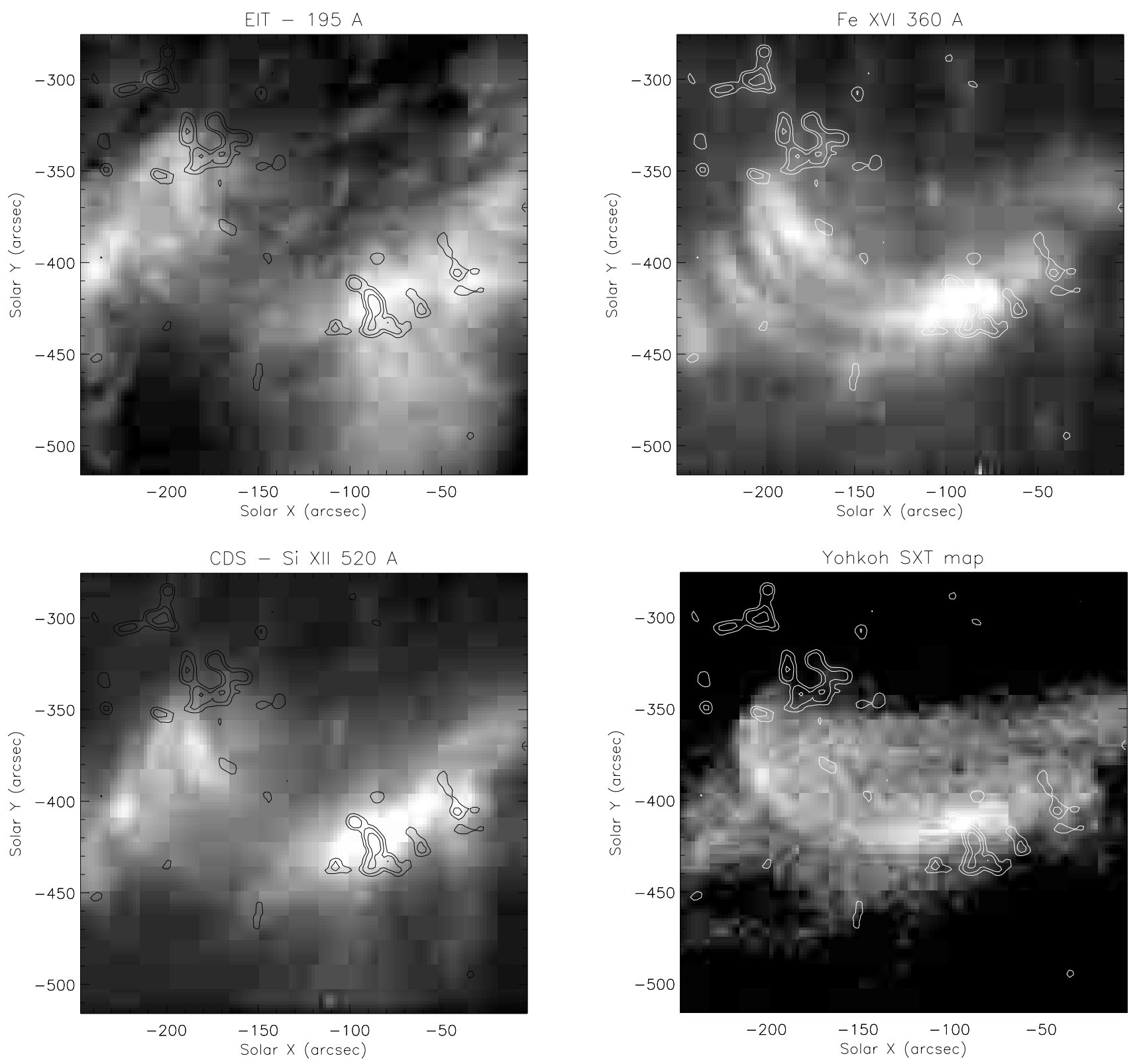

Fig. 3. Top: intensity map obtained with EIT in the $195 \AA$ channel, mainly composed by FexII intensity formed at around $1.6 \times 10^{6} \mathrm{~K}$. Bottom: intensity map obtained with CDS in the Si XII $520.67 \AA$ line. The emitting plasma has a temperature of around than $1.9 \times 10^{6} \mathrm{~K}$. Magnetic field contours from MDI are superimposed.

and 08:20 UT. Finally, MDI full disc magnetograms and intensity images with a pixel size of $1.98^{\prime \prime} \times 1.98^{\prime \prime}$ have been employed. The MDI data were recorded at 09:40 UT.

CDS raw data were cleaned and calibrated using the standard routines and calibration data available in the CDS software tree. Cosmic rays were eliminated using the "NEW_SPIKE" routine available in the CDS software. A careful analysis revealed that CDS and EIT pointings in the solar disc coordinate system are only marginally reliable. A procedure was designed to obtain more accurate pointing values. First, the EIT pointing was redetermined by comparing the solar limb position of the

Fig. 4. Top: intensity map obtained with CDS in the Fe XVI $360.76 \AA$ line, formed at around $3 \times 10^{6} \mathrm{~K}$. Bottom: intensity map obtained with the Yohkoh SXT instrument. The emitting plasma has a temperature greater than $2 \times 10^{6} \mathrm{~K}$. Magnetic field contours from MDI are superimposed.

full disc EIT image with that of the MDI full disc intensity images. Then, the images of all other instruments were transformed to the mid-time of the CDS observations (09:30 UT) by correcting for solar differential rotation, and later rebinned to the pixel size of the CDS. In the next step the CDS pointing was redetermined by cross-correlating the CDS He I $584.33 \AA$ scan (after integrating spectrally over the line profile) with the EIT He II $304 \AA$ filtergram. Finally, from all images we cut the portion which spatially corresponds to the area of the CDS raster. After this, all the sub-images cover the same area on the Sun, although context images with a larger area 
Table 1. Lines observed with CDS and used in the analysis. $\star$ : density dependent lines.

\begin{tabular}{lcccc}
\hline Ion & $\lambda(\AA)$ & $\log T$ & Detector & \\
\hline Si VIII & 314.327 & 5.93 & NIS 1 & $\star$ \\
Mg VIII & 315.039 & 5.91 & NIS 1 & \\
Fe XIII & 318.128 & 6.20 & NIS 1 & $\star$ \\
Fe XIV & 334.172 & 6.27 & NIS 1 & $\star$ \\
Fe XVI & 335.410 & 6.43 & NIS 1 & \\
Fe XII & 338.278 & 6.14 & NIS 1 & $\star$ \\
Fe XIII & 348.183 & 6.20 & NIS 1 & $\star$ \\
Ne V & 359.382 & 5.47 & NIS 1 & \\
Fe XIII & 359.642 & 6.20 & NIS 1 & $\star$ \\
& 359.842 & 6.20 & NIS 1 & $\star$ \\
Fe XVI & 360.760 & 6.43 & NIS 1 & \\
Fe XII & 364.467 & 6.14 & NIS 1 & $\star$ \\
Mg VII & 367.674 & 5.81 & NIS 1 & \\
& 367.683 & 5.81 & NIS 1 & \\
Mg IX & 368.070 & 5.98 & NIS 1 & \\
Si XII & 520.665 & 6.27 & NIS 2 & \\
Si XI & 580.907 & 6.20 & NIS 2 & \\
He I & 584.334 & 4.00 & NIS 2 & \\
O III & 599.597 & 4.96 & NIS 2 & \\
Si XI & 604.147 & 6.20 & NIS 2 & \\
O V & 629.730 & 5.39 & NIS 2 & \\
\hline
\end{tabular}

coverage were also retained. Images of the emitting region are displayed in Figs. 1 to 4.

\section{Data analysis}

\subsection{Morphology of the emitting region}

Figures 1 to 4 show that the observed active region includes several distinct structures. Plasma at around $10^{6} \mathrm{~K}$, emitting the Mg IX line, shows several loop-like structures starting from a region of enhanced magnetic field and arching out of the frame. This is visible also in the EIT-171 channel. These structures become fainter and then disappear, thus showing that their height is greater than the gravitational scale height. It is not possible to trace the other footpoint of these structures and they are not identified at other temperatures.

Hotter plasma reveals the presence of different structures, visible in Figs. 3 and 4; These are hotter, shorter, and connect directly the two regions of opposite magnetic polarity. The FexVI intensity map shows three distinct loop structures connecting the regions of opposite magnetic polarity (see Fig. 4 top); these structures are only partially visible in the EIT-195, CDS Si XII and YohkohSXT intensity maps. The intensity along them is much more uniform than in the Mg IX emitting loops, thus suggesting that their height is smaller than the gravitational scale height and the density and pressure are approximately constant. Emission from transition region lines (e.g. Ov, Fig. 1 bottom) does not show any loop-like structure, but is stronger in positions corresponding to enhanced magnetic field.
Figures 2 to 4 show that plasma at different temperatures is mostly confined in completely different structures. It is interesting to note that hotter loops such as those seen with SXT or Fe XVI do not show adjacent regions of emission from cooler coronal ions such as MgIX following the shape of the loop. This is consistent with other results from SOHO instruments, which have unexpectedely proved that the relationship between loop structures formed at different temperatures is very weak. Matthews \& Harra-Murnion (1997) have shown that loops observed with lines formed at different temperatures seem to be unrelated, and the physical characteristics and temporal evolution of Transition Region and coronal structures seem to be independent. Also, Brekke et al. (1997b) report that even small differences in line formation temperatures result in different loop structures. These simple considerations seem to suggest that loops are nearly isothermal for most of their length.

\subsection{Loop selection}

The selection of the loop shapes is of crucial importance for the quantitative comparison of the loop model described in Paper I and the present observations. This has been carried out using the intensity maps from CDS, as well as the context images provided by EIT (171, 195 and 284 channels) and Yohkoh. MDI magnetograms have been used to better identify the loop footpoints.

Unfortunately, the spatial resolution of all these instruments is rather poor, so that the emission from individual loops is contaminated by the emission from nearby structures, and the resulting image is rather confused.

However, from Fig. 4 it is possible to identify three main loop-like structures emitting in the strong FeXVI line. These structures seem to connect the two regions of opposite polarity shown by the MDI magnetogram (Fig. 1). However, an unambiguous determination of the footpoints of each of these structures is not an easy task.

If we try to identify the footpoint on the right of these loop-like structures, we can see that the two innermost of them dive into the rather confused bright area at around $\left(-90^{\prime \prime},-420^{\prime \prime}\right)$, where also other structures, formed at different temperatures and visible in the Mg IX and EIT-171 channel intensity maps, are present and are clearly unrelated to the hotter Fe XVI emitting structures. Defining the right footpoint of these two structures is nearly impossible with the present dataset. The outermost structure is fainter, but it seems to lie south of this confused blob and to terminate in the southernmost knot of magnetic field. A residual uncertainty is however left on this footpoint identification.

The left footpoint is difficult to determine for the two innermost structures, as no obvious continuation of the Fe XVI emission is seen in intensity maps from colder lines. A better identification is possible for the outermost of the three Fe XVI loop-like structures, as it matches a loop-like rising structure visible in the EIT-171 and, less clearly, in 
the EIT-195 images. This rising structure terminates directly in magnetic field enhancements. This can be more easily seen in Fig. 5, where an intensity map composed by Fe XVI and EIT-171 emission is displayed. EIT-171 intensity is displayed around the left footpoints of the Fe XVI loop-like structures.

From the above considerations, it is clear that the only loop for which a reasonable shape definition can be given is the outermost Fe XVI structure, although the right footpoint position is not well defined. So, in the rest of the present work, we will restrict our analysis to this structure only. Using the CDS intensity maps, we have selected a spatial mask defining what appears to be a reasonable approximation of the outermost loop-like structure, to be used for the quantitative comparison with the theoretical loop model of Paper I. This spatial mask, together with a combination of the FexVI and EIT-171 intensity maps, is displayed in Fig. 5.

It is important to realize that given the moderate spatial resolution of CDS, there is still a residual uncertainty on the shape of the selected loop structure around the right footpoint. The effect of this uncertainty in the diagnostic results discussed in the rest of the present paper will be described in Sects. 3.4 and 5.3.

\subsection{Temporal variability of the selected loop}

Observations from TRACE and SOHO-EIT have shown that a significant part of the active region loop structures may evolve on timescales of hours, and many of them experience even shorter-term evolution, explosive events and sudden changes in their intensity distribution. Since the theoretical model developed in Paper I is quasi-static, it is important to assess the temporal stability of the selected loop. Unfortunately, no high-cadence EIT observations have been carried out as part of JOP-54, so the temporal evolution of the selected loop structure can be checked only using periodic full-Sun images.

We have found in the EIT catalogue full-Sun EIT images obtained with the 195 channel with $\mathrm{a} \simeq 15-20 \mathrm{~min}$ cadence. These images have been used to check the stability of the selected loop. For each of these images we have calculated the running difference with the previous one, in order to have a quantitative estimate of the changes of the intensity distribution of the loop shape in the time interval between the two images. It is important to note that this running difference will be influenced by the effects of the solar rotation as well. During $20 \mathrm{~min}$, a feature at the solar surface will move $\simeq 3$ arcsec westward, so that the effect should be visible, but quite limited.

The results show that for the selected loop the differencies in the intensity rise up to a maximum of $20 \%$ of the emission along the whole of its length in only a few cases, and that for most of the considered time the differencies are of the order of, or smaller than $10 \%$. It is therefore reasonable to assume that the selected loop is quiescent.

\subsection{Density, temperature and pressure diagnostics}

The theoretical line intensity ratios for density and temperature diagnostics have been calculated using the CHIANTI database (Dere et al. 1997, 2001; Landi et al. 1999).

The selected loop has been divided into 27 subsections, each approximately $5900 \mathrm{~km}$ long. The emission observed by CDS has been averaged over each of these loop sections, so that it has been possible to measure the line intensities along the loop, and through them it has been possible to determine the temperature and density along the length of the structure.

In order to analyze radiation emitted from the loop structure only, it has been necessary to subtract the background radiation. The choice of the background to be subtracted is crucial in this kind of study, especially for instruments with moderate spatial resolution like CDS, since the presence of any structures close to the selected loop is likely to contaminate its emission. In the present work, the background to be subtracted to each of the subsections composing the selected loop has been determined by averaging the emission of the surrounding pixels closest to each of the subsections. In other words, its own background has been associated to each loop subsection. This choice has allowed us to take into account the variation of the background emission along the loop shape, given by the presence of brighter features in the field of view.

Since for many lines the emission of the background constituted a significant portion of the total observed intensity, the resulting uncertainties are rather high in some cases (up to $40 \%$ ).

\subsubsection{Density diagnostics}

Several density sensitive lines are available in the CDS data and are listed in Table 1. Lines of Fe XII (364.47 $\AA$ and $338.28 \AA$ ) and Fe XIII (359.64 $\AA+359.84 \AA$ and $348.18 \AA$ ) have been considered. The Ne v blending line at $359.3 \AA$ has been subtracted from the $359.64 \AA$ and $359.84 \AA$ lines to compute Fe XIII line ratios. The Fe XIII $318.13 \AA$ line has not been used because ratios involving this line provide unusually high values of the electron density (Young et al. 1998).

Several authors have found problems with Fe XII lines, since they were found to provide higher density values than the other ions formed at similar temperatures (Brosius et al. 1996, 1998, 1999; Landi \& Landini 1997, 1998). Binello et al. (2001) have shown that these problems are due to incompleteness in the atomic model for FeXII, and so we have exclusively used FexIII lines for density diagnostics.

The electron density shows, within the uncertainties, a constant behaviour along the whole loop length, with an average value of $\log N_{\mathrm{e}}=9.3\left(\mathrm{~cm}^{-3}\right)$. The constant density implies that the loop total height is still smaller than the gravitational scale height. Since the distance between the footpoints of the loop observed in the intensity maps 


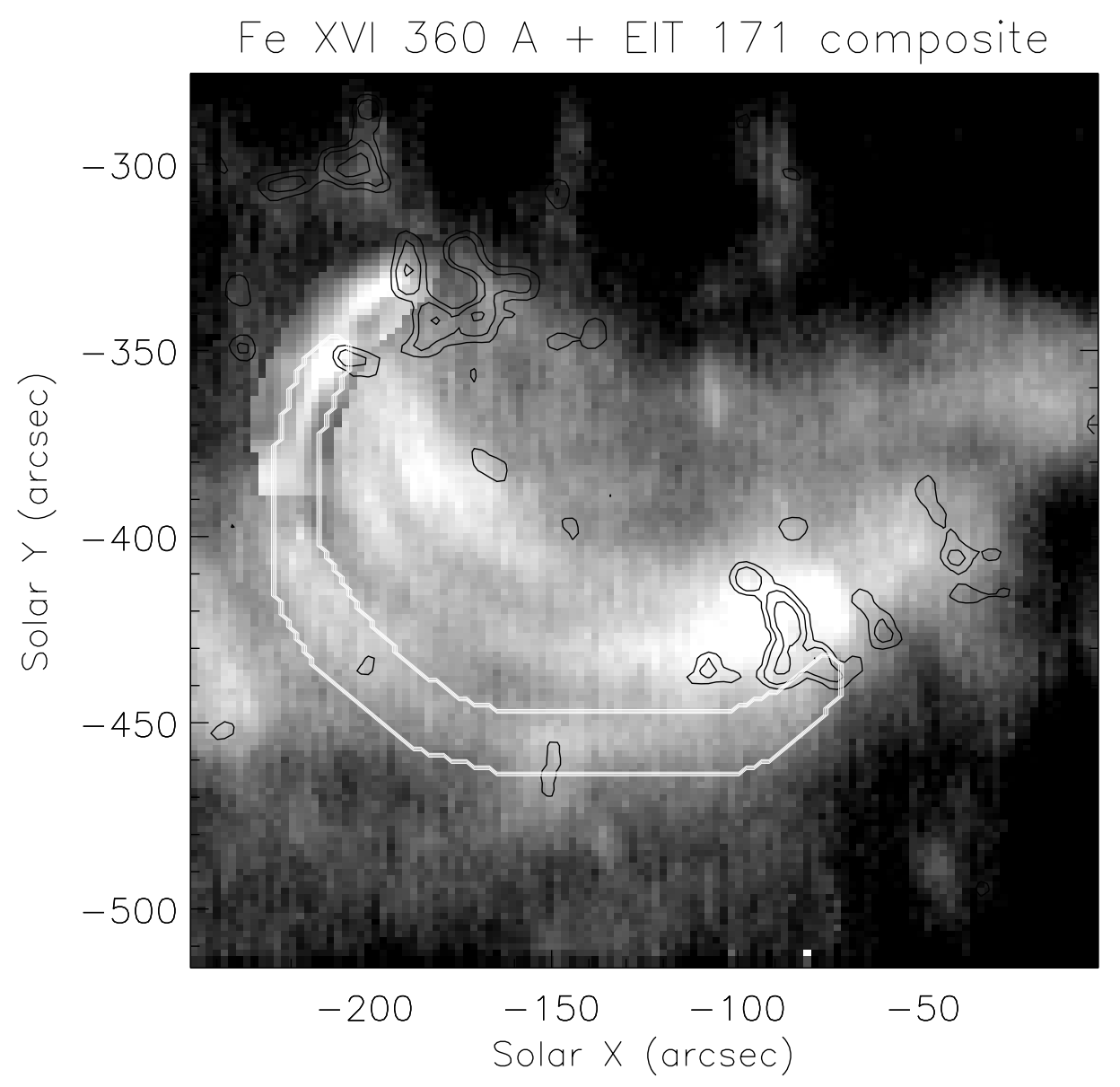

Fig. 5. Composite intensity map obtained with CDS in the Fexvi $360.76 \AA$ line, and the EIT-171 channel. The shape of the selected loop is overplotted. Magnetic field contours from MDI have also been displayed to show the loop footpoints. The EIT emission has been used to show the position of the left footpoint of the selected loop, and is visible due to the different background intensity.

is of the order of, or greater than, the gravitational scale height (around $9 \times 10^{4} \mathrm{~km}$ ), the loop either is inclined relative to the vertical, or departs from a quasi-semicircular shape (see also Sect. 3.5).

\subsubsection{Temperature diagnostics}

Temperature measurements are performed through the Fe XVI/Fe XIV line ratio, taking into account the density sensitivity of the Fe XIV spectral line. This ratio is the most sensitive to high temperatures among the ratios available in the present dataset; moreover Fe XIV and FeXVI provide the only CDS lines that clearly show the whole of the three selected loop structures. The results are displayed in Fig. 6.

The electron temperature of all loops exhibits a very flat shape for most of the length of the loop. This nearly constant behaviour has already been noted by Aschwanden et al. (1999, 2000a) and Lenz et al. (1999). The temperature results have been obtained adopting the ion fractions of Arnaud \& Raymond (1992). However, changing the ion fractions dataset might cause changes in the measured values of the electron temperature, due to the importance of ion fractions for temperature diagnostics and to the fact that different authors may provide significantly different ion fractions in the literature. This possibility has been explored by Gianetti et al. (2000) and Allen et al. (2000). For this reason, a check has been performed by determining the electron temperature adopting also the ion fractions from Shull \& Van Steenberg (1982), Arnaud \& Rothenflug (1985) and Mazzotta et al. (1998). The results obtained with all these different theoretical datasets show a variability of $\pm 8 \%$ around the values obtained using the Arnaud \& Raymond (1992) ion fractions. As this variability is greater than the experimental errors, it has been taken as the uncertainty of the measured temperature values.

The use of the Fe XVI/Fe XIV line ratio might generate a bias of the measured electron temperatures, by forcing the resulting electron temperature to be in the temperature range $6.2 \leq \log T \leq 6.4$, where these two ions are principally formed. In order to assess the effects of this bias, we have carried out, for each of the loop subsections, an emission measure analysis, described in the following. Further details of this emission measure analysis can be found in Allen et al. (2000) and Landi et al. (2001a). 
The flux of an optically thin emission line observed at distance $d$ can be written as

$I_{j i}=\frac{1}{4 \pi d^{2}} \int_{V} G_{j i}\left(T, N_{\mathrm{e}}\right) N_{\mathrm{e}}^{2} \mathrm{~d} V$

where $N_{\mathrm{e}}$ is the electron density and $G_{j i}\left(T, N_{\mathrm{e}}\right)$ is the Contribution Function of the emitting line. If the electron density $\left(N_{\mathrm{e}}\right)$ and temperature $\left(T_{\mathrm{c}}\right)$ are constant along the line of sight, we have

$I_{j i}=\frac{G_{j i}\left(T_{\mathrm{c}}, N_{\mathrm{e}}\right)}{4 \pi d^{2}}<E M>\quad<E M>=\int_{V} N_{\mathrm{e}}^{2} \mathrm{~d} V$

where $\langle E M\rangle$ is the Emission Measure of the plasma. In this case the Emission Measure can be directly evaluated as:

$<E M>=4 \pi d^{2} \frac{I}{G\left(T_{\mathrm{c}}, N_{\mathrm{e}}\right)}$.

This quantity is the same for all the observed lines. The diagnostic method consists of calculating the function $<E M(T)>$ defined as

$<E M(T)>=\frac{I}{G\left(T, N_{\mathrm{e}}\right)}$

as a function of electron temperature, using the observed intensities $I$ of each observed line and a value of the electron density derived from line ratio techniques. Since

$<E M\left(T_{\mathrm{c}}\right)>=\frac{1}{4 \pi d^{2}}<E M>$

when all the $<E M(T)>$ curves are displayed in the same plot as a function of temperature, all these curves will intersect in a common point at $\left(T_{\mathrm{c}}, \frac{1}{4 \pi d^{2}}<E M>\right)$. The common crossing point directly determines the plasma temperature $T_{\mathrm{c}}$ and $\langle E M\rangle$. When applied to each subsection, this analysis allows to check whether each of the loop subsections is characterized by a single temperature, to measure its value and the value of the emission measure.

In each subsection, only the lines whose intensity was greater than zero were used; in all subsections, a common crossing point was identifiable within the uncertainties, and the electron temperature $T_{\mathrm{c}}$ and $\langle E M\rangle$ were evaluated. Results confirm the temperatures measured with the Fe XVI/Fe XIV line ratio within the uncertainties. Only in the two subsections overlying the footpoints of the loop the plasma showed a clearly non-isothermal behaviour and no common crossing point existed, consistently with the fact that in those subsections the line of sight crosses the footpoint regions, where the temperature gradient is largest and the plasma is not isothermal.

The measurements of the $\langle E M\rangle$ values are discussed in Sect. 3.7.

\subsubsection{Pressure diagnostics}

From the measured values of electron temperature and density it was possible to determine the electron pressure $p_{\mathrm{e}}$ along the loop structure. The total plasma pressure is then calculated as $1.9 \times p_{\mathrm{e}}$, assuming a fully ionized
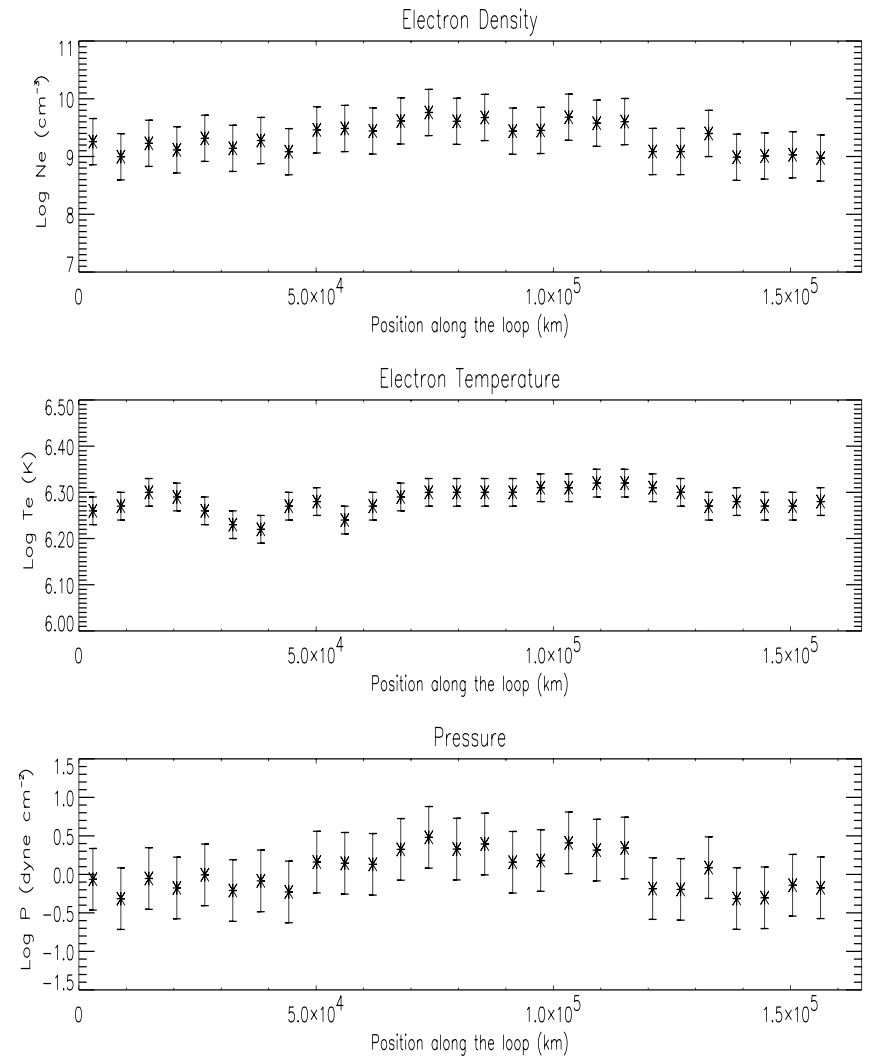

Fig. 6. Plasma diagnostics for the selected loop in Fig. 5.

plasma of standard composition. The total pressure is also displayed in Fig. 6. One can see that a constant pressure value satisfies, within the experimental uncertainties, all the subsections, with the exception of the last two, corresponding to the region closest to the right footpoint.

The uncertainty in the position of the right footpoint of the selected loop results in some additional uncertainty in the measurements of temperature, density and pressure of the last two loop subsections on the right in Fig. 6 . Diagnostics in these two locations should therefore be treated with some caution.

These results allow us to use the contant-pressure loop model described in Paper I, to be compared with the present observations. The average pressure value is $\simeq 0.9$ dyne $\mathrm{cm}^{-2}$, and is compatible with the values reported in the literature (see Aschwanden et al. 2000a and references therein). The variation of each individual value around the mean is due to the uncertainties in the electron density.

\subsection{Loop geometry and pressure scale height}

The temperature measured in the loop structure is around $1.9 \times 10^{6} \mathrm{~K}$, and this allows us to determine the pressure scale height for the coronal part of the loop to be around $9 \times 10^{4} \mathrm{~km}$.

In order to compare this scale height with the geometrical height of the loop it is necessary to make some assumption on the geometry of the loop structure itself. 
Figure 5 suggests that the selected loop can be approximated with a semicircular structure inclined relative to the vertical. If we make this assumption then simple geometrical considerations show that the selected loop is nearly parallel to the solar surface (angle smaller than $18^{\circ}$ ). Measuring the distances between the footpoints of each loop from Fig. 5 and considering them as the diameter of the semicircular structure, it is possible to determine the maximum height reached by the loop structure: $<17000 \mathrm{~km}$. This means that for the selected loop the expected maximum pressure difference is smaller than 0.2 dex. Since the uncertainty in the pressure measurements is greater than 0.2 dex, the constant pressure assumption is verified within the experimental uncertainties.

The loop cross-section at coronal temperatures has been determined from the mask displayed in Fig. 5, and its value has been corrected for the CDS spatial resolution, taken as 6 arcsec along in the E-W direction and 8 arcsec in the N-S direction (Pauluhn et al. 1999). Assuming that the cross-section is circular, its resulting value is $5.8 \times 10^{17} \mathrm{~cm}^{2}$. However, the CDS spatial resolution does not allow us to distinguish between monolithic loops and loops composed of many thin strands, as suggested by TRACE (Transition Region And Coronal Explorer, Handy et al. 1999).

\subsection{Velocity fields}

In order to check whether the selected loop structure harbours velocity fields strong enough to have consequences for the theoretical loop model, measurements have been made of spectral line positions both inside the spatial mask of the selected loop, and in the rest of the map. Spectral line positions have been measured in each pixel inside the mask after the subtraction of the background spectrum. Spectral lines have been assumed to have a Gaussian shape and have been fitted using the ADAS602 fitting routine (Brooks et al. 1999); both coronal and transition region lines have been considered.

Results show that speeds along the loop shapes are always smaller than $15 \mathrm{~km} \mathrm{~s}^{-1}$, while the sound speed for the loop plasma (with $T \simeq 1.9 \times 10^{6} \mathrm{~K}$ ) is around $200 \mathrm{~km} \mathrm{~s}^{-1}$. These measurements verify that the quasi-static assumption adopted in Paper I is valid for the present structures.

It is important to note that spectral line positions allow only line-of-sight component of velocities to be determined, so that in the central part of the loops, where the direction of the flow is nearly perpendicular to the line of sight, the real speed can be higher than this value. The low value of the plasma speed at the footpoints indicates, however, that it is unlikely that flows in the top portion of each loop can affect the theoretical model.

\subsection{Filling factor of the selected loop}

Once the electron density and temperature and emission measure $E M$ are determined along the loop shape, it is possible to calculate the filling factor of each of the subsections composing the structure. The plasma volume can be evaluated using the assumption of circular cross-section and the linear dimension of each subsection measured on the intensity map. This, together with the measured electron density, allows us to determine the subsection emission measure as

$E M_{i}^{\text {pred }}=N_{\mathrm{e}_{i}}^{2} \times V_{i}$.

This value can be compared with the Emission Measure value measured from the observations in Sect. 3.4.2 and the loop filling factor can be derived as

$f f=\frac{E M_{i}^{\mathrm{obs}}}{E M_{1}^{\mathrm{pred}}}$.

The resulting filling factor, averaged over all the subsections, is

$f f=0.33 \pm 0.05$.

The uncertainties on this value is given as the standard deviation of the average, but it is important to note that the real uncertainties on each subsection filling factor are much larger, due to the uncertainties in the electron density, so that each of the $f f$ values, as well as their average, should be considered only as a rough estimates. However, this result indicates that the filling factor of this coronal active region loop is smaller than, but not far from, unity.

\section{Comparison with the theoretical model}

In this section, the comparison between the observations and the loop model developed in Paper I is described. The comparison is carried out examining four cases:

- I - the diagnostic procedure coming from the standard model is applied to the observations (Sect. 4.2);

- II - an isothermal region is added on top of the loop model and the diagnostic technique is applied again (Sect. 4.3);

- III - The assumption of a thermally-insulated loop underlying the model is relaxed, and the diagnostic technique is applied to the loop model, without an isothermal region at the top (Sect. 4.4);

- IV - The isothermal region is added at the top of the non-thermally-insulated loop model (Sect. 4.5).

In the case of a non-thermally-insulated loop, we have run the model adopting a scaled conductive flux at the base of our model $\left(T=2 \times 10^{4} \mathrm{~K}\right)$ of $f_{0}=1.5$ (see Paper I). This corresponds to values in the range 1.5$2.0 \times 10^{6}$ erg $\mathrm{cm}^{-2} \mathrm{~s}^{-1}$, compatible with Oluseyi et al. (1999).

The comparison involves line intensities observed with CDS. Since the diagnostic technique must be applied to the intensities emitted by the whole loop, the spectra observed by CDS have been summed over the loop mask displayed in Fig. 5. The background radiation, evaluated as described in Sect. 3.4 and then summed over all the loop subsections, has been subtracted from the resulting total spectrum. 


\subsection{Overview of the method}

The diagnostic method is described in detail in Paper I. Here only a very brief outline will be given.

According to the loop model in Paper I, we can write the flux received at Earth at distance $d$ in an optically thin spectral line $j$ emitted by a loop as

$F_{j}=\frac{S_{M} p_{0}}{4 k^{2}} T_{\mathrm{M}}^{\star} \sqrt{\frac{A}{C}} \frac{1}{4 \pi d^{2}} \int G_{j}\left(T, N_{\mathrm{e}}\right) \frac{t^{1 / 2}}{w} \sigma^{2}(t) \mathrm{d} t$

where $G_{j}\left(T, N_{\mathrm{e}}\right)$ is the Contribution Function of the line $j ; t, w, \sigma$ are the scaled temperature, conductive energy and cross-section of the loop, $A=0.92 \times$ $10^{-6} \mathrm{~K}^{-3.5} \mathrm{erg} \mathrm{cm}^{-1} \mathrm{~s}^{-1}, C=2 \times 10^{12} \mathrm{~K}^{2.5} \mathrm{~cm}^{3} \mathrm{erg}^{-1} \mathrm{~s}^{-1}$, $S_{M}$ is the maximum cross-section of the loop, $p_{0}$ is the total loop pressure, $T_{\mathrm{M}}^{\star}$ is the temperature at which radiative losses equal the energy input in the observed loop (all the quantities are in cgs units). It is possible to define the ratio $R\left(T_{\mathrm{M}}\right)$ as

$R\left(T_{\mathrm{M}}\right)=\frac{F_{j}^{\mathrm{obs}}}{J_{j}\left(T_{\mathrm{M}}\right)}$

where $F_{j}^{\text {obs }}$ is the observed line flux integrated over the whole loop shape, and $J_{j}\left(T_{\mathrm{M}}\right)$ is a quantity given by

$J_{j}\left(T_{\mathrm{M}}\right)=T_{\mathrm{M}} \int_{T} G_{j}(T) \operatorname{DEM}\left(\frac{T}{T_{\mathrm{M}}}\right) \mathrm{d} T$

$T_{\mathrm{M}}$ is the temperature at which radiative losses equal energy input in the theoretical model. The $R\left(T_{\mathrm{M}}\right)$ function can be evaluated for each observed line, and displayed on the same plot as a function of $T_{\mathrm{M}}$. If the loop model is correct, it is possible to show that when $T_{\mathrm{M}}=T_{\mathrm{M}}^{\star}$ all the curves have the same value and meet in the point

$$
\left(T_{\mathrm{M}}^{\star}, \frac{S_{\mathrm{M}} p_{0}}{4 k^{2}} \sqrt{\frac{A}{C}} \frac{1}{4 \pi d^{2}}\right) .
$$

From this crossing point it is possible to simultaneously measure both the value of $T_{\mathrm{M}}^{\star}$ (and hence the loop top temperature) and of $p_{0} S_{\mathrm{M}}$.

In the present work, the value of $T_{\mathrm{M}}^{\star}$ determined from the crossing point is used to determine the loop-top temperature and the loop half-length using the relations

$T_{\text {top }}=c\left(T_{\mathrm{M}}\right) T_{\mathrm{M}}$

$L_{\mathrm{loop}}=r\left(T_{\mathrm{M}}\right) \sqrt{\frac{A}{C}} \frac{T_{\mathrm{M}}^{3}}{p_{0}}$

where $r\left(T_{\mathrm{M}}\right)$ and $c\left(T_{\mathrm{M}}\right)$ are discussed in detail in Paper I.

The loop top temperature determined from the model can be compared with the results obtained with the emission measure analysis and line ratios.

The measured $p_{0} S_{\mathrm{M}}$ value is used to determine the loop maximum section $S_{\mathrm{M}}$ by using the pressure $p_{0}$ determined through line ratios; the resulting value can be compared with observations.
Also, since the loop shape affects mostly the coldest lines in the dataset, the loop shape parameters $\left(S_{\mathrm{M}} / S_{\mathrm{m}}\right.$ and $n$, see Paper I) are determined as those giving the best agreement between all the lines in the dataset. It is important to note that the greatest effects of the crosssection variability are found for the colder lines (i.e. $\mathrm{OV}$, Mg VII-IX, Si VIII), and they decrease as the temperature of formation of the line increases. Therefore, the best diagnostic in the present active region dataset is given by the O v line, while the sensitivity to loop shape variations of the other $\mathrm{Mg}$, Si and Fe lines is more and more limited as the emitting ions are formed at higher temperatures.

In the present work, line emissivities have been taken from the CHIANTI database, with the exception of very few lines not yet included in CHIANTI. Element abundances have been taken from Feldman (1992), and ion fractions are taken from Arnaud \& Rothenflug (1985) for all elements but iron, which comes from Arnaud \& Raymond (1992).

A few lines have been discarded from the diagrams, since they badly missed the crossing point:

- The Sixi $520.67 \AA$ line showed a too high theoretical flux. This behaviour has already been noted in the past: Feldman et al. (1998) report that all Li-like ions show a systematic excess in theoretical emissivities relative to all the other ions of the other isoelectronic sequences; similar results have also been found by Judge et al. (1995);

- Young et al. (1998) and Binello et al. (2001) suggested that the Fe XII line at $338.278 \AA$ is blended with an unidentified line, and this is confirmed here as this line shows an excess of observed flux;

- The Fe XIII $318.13 \AA$ line is rejected as it is suspected to have some problems (Young et al. 1998);

- both the Si XI intercombination lines at $580.91 \AA$ and $604.15 \AA$ show a too high observed flux. This behaviour is possibly due to atomic physics problems, as indicated by Landi et al. (2001b);

- He I is not used in the present work since its lines are believed not to be optically thin (see Andretta \& Jones 1997 and references therein), so that the diagnostic technique cannot be applied to them.

In all the following applications of the diagnostic technique, these lines will not be used. The results of the comparison in all the four cases considered are reported in Table 2. In the following each of them will be discussed in more detail.

\subsection{Case I: Loop diagnostics using the standard model}

The application of the diagnostic technique to the selected loop is illustrated in Fig. 7. It is possible to see that a crossing point for all the $R\left(T_{\mathrm{M}}\right)$ curves is rather well defined.

From Table 2 it follows that the agreement between theoretical and observed loop-top temperature values is reasonable, with theoretical temperatures only slightly too 


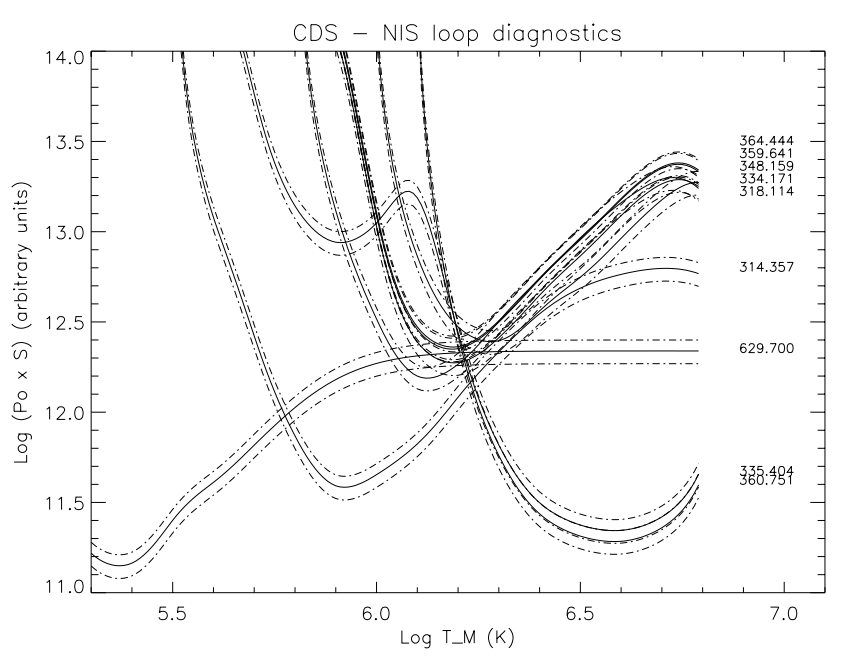

Fig. 7. Application of the loop diagnostic technique for the selected loop structure to Case I: no isothermal region has been added at the top of the loop. The theoretical model is assumed to be thermally insulated at the base.

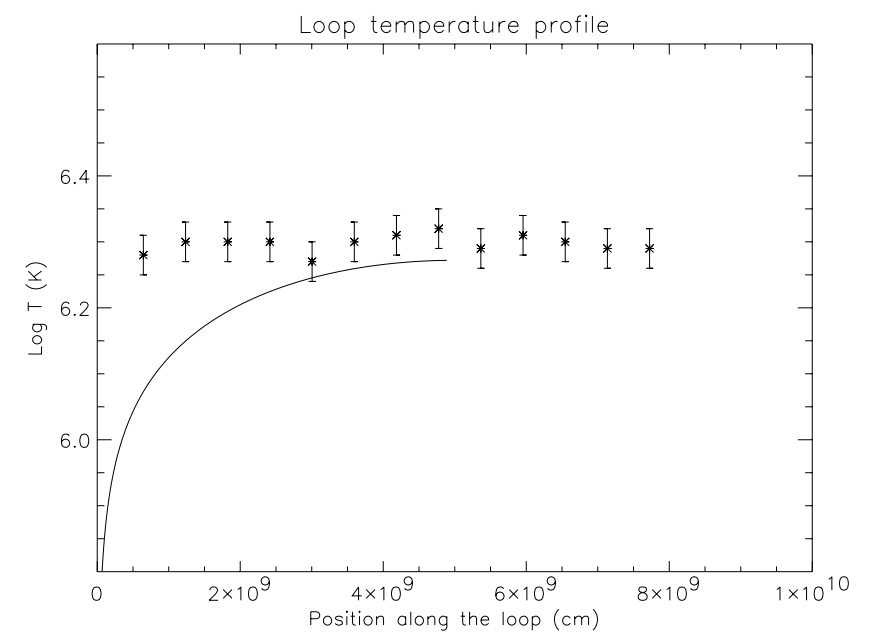

Fig. 8. Theoretical temperature profile of the selected loop, from the footpoint to the top. The overplotted measurements are the temperature values determined with the line ratio technique. Case I: no isothermal region has been added at the top of the loop. The theoretical model is assumed to be thermally insulated at the base.

low. Agreement between the measured and the predicted loop cross-section radius is within the uncertainties.

There are, however, two main problems in the results reported in Table 2: the total loop lengths and the loop temperature profiles predicted by the model.

The disagreement found between observed and predicted loop lengths indicate that some problem is present in the model. The loop length predicted by the loop scaling law in Eq. (14) is too small.

The comparison of the measured and the observed temperature profiles of the loop reveals an even greater difficulty of the model. The measured and predicted temperature profiles are displayed in Fig. 8. The projection effects due to the inclination of the loop relative to the solar surface are small, as the inclination angle is smaller than $18^{\circ}$ (see Sect. 3.5) and for this reason have not been accounted for in Fig. 8. Obviously the experimental temperature profile of the loop is much more constant than the theoretical one. The diagnostic results displayed in Fig. 8 confirm the findings of Neupert et al. (1998), Aschwanden et al. (1999, 2000a), Lenz et al. (1999), and indicate that the coronal loops studied here are much more isothermal than the present model predicts.

The main constraint on the loop shape is given by the colder lines (O v, Si viII, Mg VII-IX). Hot coronal lines are less affected by such geometrical effects because they are formed where the loop cross-section is closer to the maximum value $S_{\mathrm{M}}$. On the contrary, even small changes in the $S_{\mathrm{M}} / S_{\mathrm{m}}$ ratio and in the parameter $n$ have huge effects on these colder lines, because the loop shape strongly depends on these parameters for temperatures similar to the formation temperature of these ions. The measured $S_{\mathrm{M}} / S_{\mathrm{m}}$ ratio allows us to determine the size of the footpoint region of the loops: these appear to be of the same size as, or smaller than the pixel size and hence definitely smaller than the instrumental point spread function.

However, since the colder ions emission comes from the footpoint regions, whose identification is rather difficult, the determination of the loop shape parameters has a rather large uncertainty. This is further discussed in Sect. 5.3. However, the effect on the determination of the crossing point is much more limited, since this is mainly given by the hot coronal lines, whose main contribution is given by the hottest part of the loop, which is reasonably well defined.

These results lead to consider the possibility of adding an artificial isothermal region at the top of the model loop, and to include it when applying the diagnostic technique and comparing the model with the observations.

\subsection{Case II: Loop diagnostics when adding an isothermal section to the standard model}

In order to apply the diagnostic procedure in the presence of an isothermal region at the loop top, it is necessary to subtract the contribution of the latter from the total line flux coming from the loop. Once this contribution is eliminated, the procedure is applied to the resulting line intensities in the same way as in the previous Section.

In order to calculate the emission of the isothermal region, it is necessary to calculate its Emission Measure and the Contribution Function. Therefore the electron density, temperature and geometry of this region must be known. The isothermal section has been assumed to be cylindrical, with cross section equal to the $S_{\mathrm{M}}$ measured from the maps. $T_{\mathrm{e}}$ and $N_{\mathrm{e}}$ have been taken as the average of the line ratio results in the selected isothermal region. The choice of the length of the isothermal region is somewhat arbitrary; to overcome this ambiguity we have repeated the study adopting several different lengths, so that a more thorough investigation of the effect of the presence of the isothermal region could be done. An example, obtained 
Table 2. Comparison between theoretical and observed physical properties of the selected loops. The cross-section radius $R$ and the loop total length $L$ values are in $10^{4} \mathrm{~km}, p_{0}$ is in dyne $\mathrm{cm}^{-2}, T$ is in K. See Paper I for a definition of $\mathrm{c}\left(T_{\mathrm{M}}\right)$.

\begin{tabular}{ccccccccccc}
\hline Case & $\frac{S_{\mathrm{M}}}{S_{\mathrm{m}}}$ & $n$ & $\mathrm{c}\left(T_{\mathrm{M}}\right)$ & $p_{0}^{\text {meas }}$ & $\log T_{\max }^{\text {meas }}$ & $\log T_{\max }^{\text {th }}$ & $L^{\text {meas }}$ & $L^{\text {theor }}$ & $R^{\text {meas }}$ & $R^{\text {th }}$ \\
\hline \hline & & & & & & & & & & \\
I & 15 & 1.60 & 1.14 & $0.90 \pm 0.20$ & $6.31 \pm 0.03$ & $6.28 \pm 0.02$ & $15.9 \pm 1.5$ & $9.8 \pm 4.1$ & $4300 \pm 1100$ & $5200 \pm 1500$ \\
II & 15 & 1.60 & 1.15 & $0.90 \pm 0.20$ & $6.31 \pm 0.03$ & $6.26 \pm 0.03$ & $15.9 \pm 1.5$ & $14.0 \pm 3.5$ & $4300 \pm 1100$ & $5000 \pm 1700$ \\
III & 3 & 1.40 & 1.51 & $0.90 \pm 0.20$ & $6.31 \pm 0.03$ & $6.28 \pm 0.03$ & $15.9 \pm 1.5$ & $5.3 \pm 2.6$ & $4300 \pm 1100$ & $6600 \pm 2700$ \\
IV & 3 & 1.40 & 1.66 & $0.90 \pm 0.20$ & $6.31 \pm 0.03$ & $6.29 \pm 0.03$ & $15.9 \pm 1.5$ & $15.0 \pm 2.5$ & $4300 \pm 1100$ & $5700 \pm 1600$
\end{tabular}

by adopting a selected length is $6.3 \times 10^{9} \mathrm{~cm}$, is displayed in Figs. 9 and 10. The physical parameters obtained from the model in this particular case are reported in Table 2 (Case II), along with the model predictions.

Table 2 reveals that the loop length shows reasonable agreement between the model predictions and the observations. Also, the predicted radius of the loop cross section is in good agreement with observations, while the predicted loop top temperature is lower than in Case I, although it still agrees with the intensity ratio value within the uncertainties. However, the temperature profile of the loop still remains problematic. Figure 10 displays the observed and theoretical temperature profiles of the selected loop. It shows that the loop model plus the isothermal region at its top is able to satisfactorily reproduce the observed temperature values only in the hottest part of the loop. The theoretical model predicts a too smooth decrease of the electron temperature near the footpoints. We have repeated this study by adopting a several different choices of the isothermal loop length, but the results do not change: the predicted temperature decrease along the loop is still too smooth in all cases. In addition, choosing too large values of the isothermal loop length causes the predicted loop cross-section to disagree with the observations. Hence, adding an isothermal region at the loop top solves only one of the two problems of the "standard" loop models.

It is also important to note that the uncertainty in the definition of the right footpoint of the selected loop has now a larger impact on the diagnostic result. This is due to the fact that a large portion of the coronal part is no longer contributing to the observed intensities analyzed with this method, so that these are now more sensitive to changes in the loop footpoint choice. Also, the effects on the loop shape definition are unchanged from the previous case.

\subsection{Case III: Loop diagnostics with non-thermally insulated loops}

Results of the procedure applied to this theoretical model are shown in Figs. 11 and 12 and the main loop properties are summarized in Table 2 (Case III). The presence of a non-negligible conductive flux at the base of the model $\left(F_{0} \simeq 1.5-2.0 \times 10^{6} \mathrm{erg}^{\mathrm{cm}^{-2}} \mathrm{~s}^{-1}\right)$ has two important

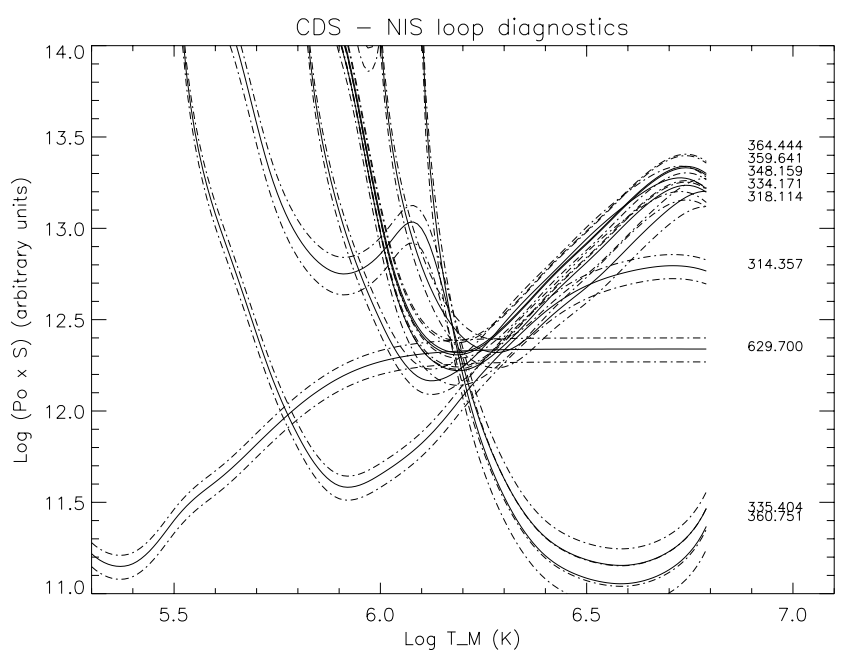

Fig. 9. Application of the loop diagnostic technique for the selected loop structure. Case II: an isothermal region has been added at the top of the loop. The theoretical model is assumed to be thermally insulated at the base.

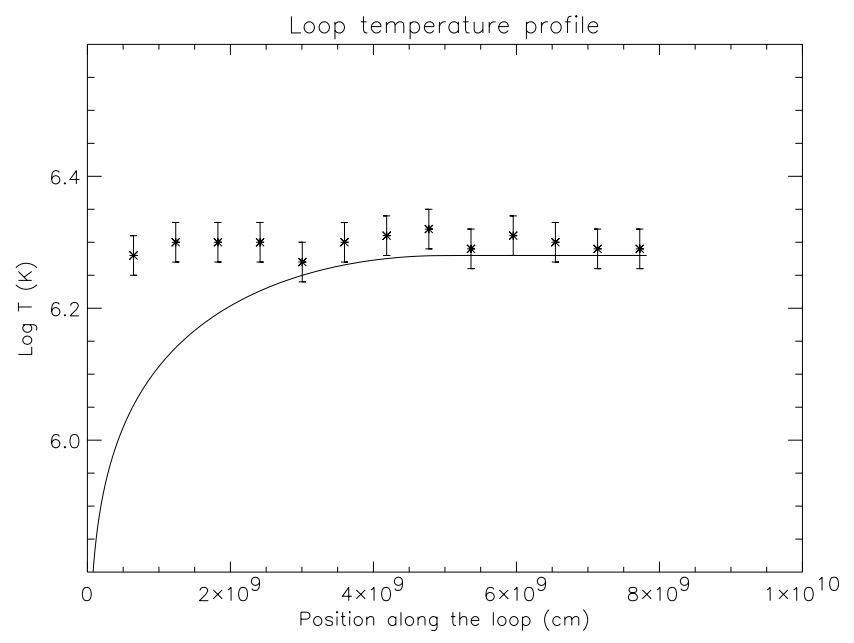

Fig. 10. Same as Fig. 8, for Case II: an isothermal region has been added at the loop top to reproduce the coronal region of the loop. The theoretical model is assumed to be thermally insulated at the base.

consequences: it changes the values of the fitted parameters of the loop shape, and lowers the value of $T_{\mathrm{M}}$, the temperature at which the input energy equals the energy lost by radiation. 
The first effect is most important on the cooler lines of our dataset; they require the $\frac{S_{\mathrm{M}}}{S_{\mathrm{m}}}$ value to decrease relative to the thermally-insulated case.

The lower value of $T_{\mathrm{M}}$ makes the problem of the theoretical loop length even worse. It is possible to see that the theoretical loop length reported in Table 2 and in Fig. 12 is much shorter than in the thermally-insulated case. Also, the predicted cross-section radius of the loop is substantially increased, although the uncertainties are large enough to reach the observed value. The loop top temperature is similar to the observed value.

These results show that a standard loop model, with the assumption of a non-negligible conductive flux at the loop base, still is not able to reproduce the observations. Choosing lower $F_{0}$ values brings the model toward the standard model, which still provides too small theoretical loop lengths; higher $F_{0}$ values than adopted cause the loop length to be even shorter, and the loop cross-section to be too large.

It is important to note however that the presence of a non-negligible $F_{0}$ causes the temperature gradient to be much sharper at low heights, so that coronal temperatures are reached earlier than in the standard model and the temperature profile is closer to the observed one at low temperatures. This is an important step in the right direction.

Finally, the effects of the uncertainties in the right footpoint of the loop are similar to those discussed in Case I (Sect. 4.2).

\subsection{Case IV: Loop diagnostics of non-thermally insulated loops with an added isothermal section}

As with the thermally-insulated model, we have inserted an isothermal region on top of the loop model, while retaining the assumption of non-negligible $F_{0}$. As for the thermally-insulated case, we have adopted several different values for the length of the isothermal section. The other parameters of the isothermal section are the same as in Sect. 4.3. The diagnostic plot and the temperature profiles are shown in Figs. 13 and 14, and the loop properties are summarized in Table 2 (Case IV) for the case where the isothermal section length is $1.1 \times 10^{10} \mathrm{~cm}$, corresponding to the loop segments whose temperature is equal to the loop-top temperature within the uncertainties.

In this case, it is possible to see that the temperature profile of the loop is in agreement with the observations. The portion of the loop covered by the non-isothermal region, given by the model, is very short (around $20000 \mathrm{~km}$ on each side) and nearly the whole length is filled by the isothermal section. The temperature gradient is very steep at the base of the loop. This is caused by the large conductive flux assumed at the loop base. Agreement with the other physical quantities that characterize the loop is also found. However, since the temperature gradient near the loop footpoints is larger than in the thermally insulated case, agreement is found only for isothermal lengths larger

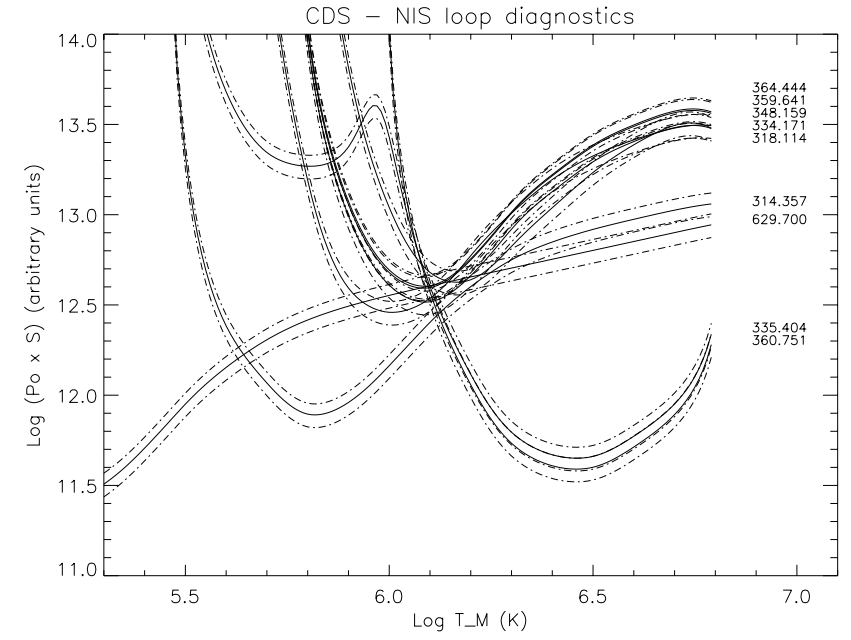

Fig. 11. Application of the loop diagnostic technique for the selected loop structure. Case III: no isothermal region has been added at the top of the loop. The theoretical model is assumed not to be thermally insulated at the base.

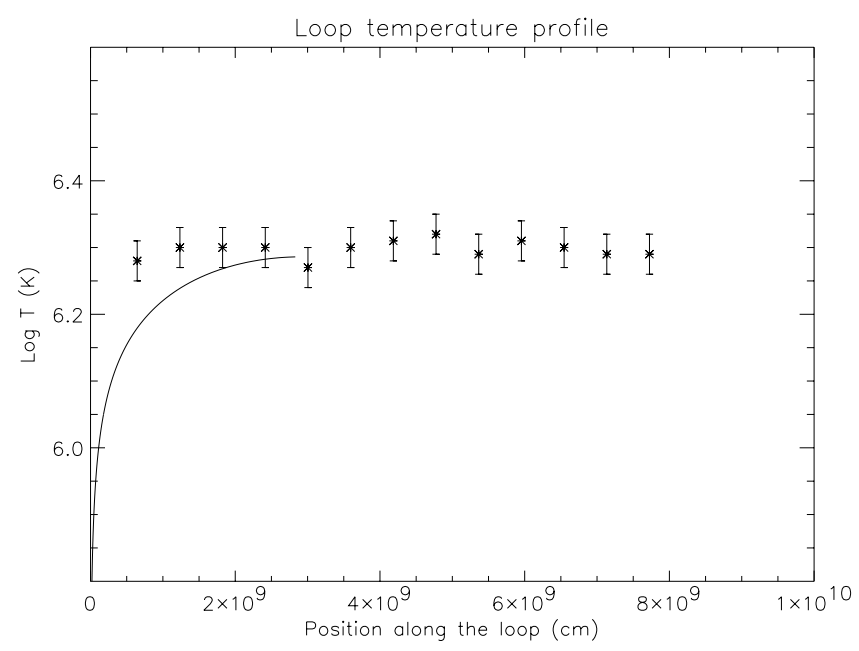

Fig. 12. Same as Fig. 8, for Case III: no isothermal region has been added at the loop top. The theoretical model is assumed not to be thermally insulated at the base.

than in the thermally insulated case, because for too short isothermal lengths the total predicted loop length is too short.

It is important however to note that in the present case the effect of the uncertainty in the selection of the right loop footpoint is at its highest, as a larger isothermal section is removed from the calculation of the line intensity to be used with the loop diagnostic technique.

\section{Discussion}

The comparisons carried out in Sect. 4 have shown that, on its own, a quasi-static standard model is not able to reproduce the observations, irrespective of whether we assume the loop to be thermally insulated or not. This result seems to hold even if we allow for the uncertainty in the 


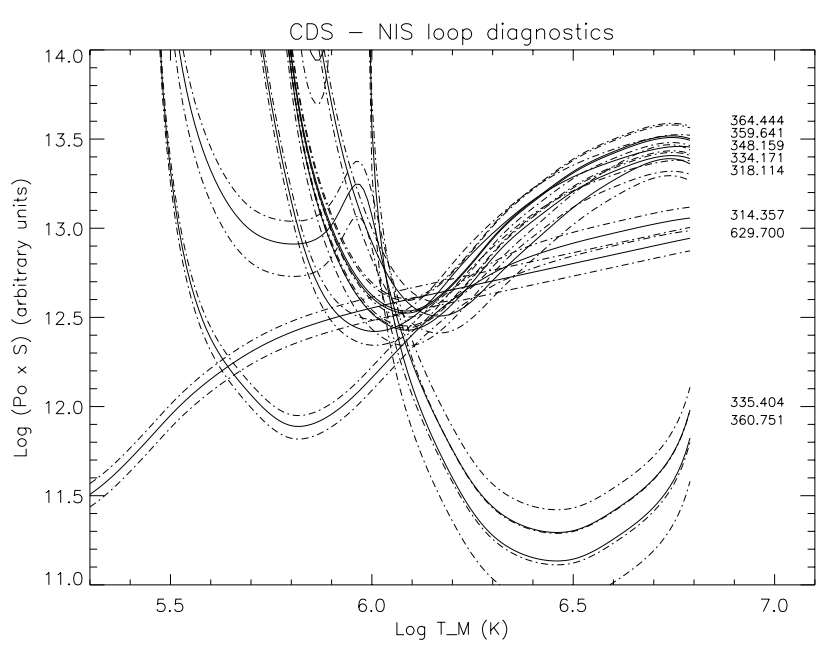

Fig. 13. Application of the loop diagnostic technique to the selected loop structure. Case IV: an isothermal region has been added at the top of the loop. The theoretical model is assumed not to be thermally insulated at the base.

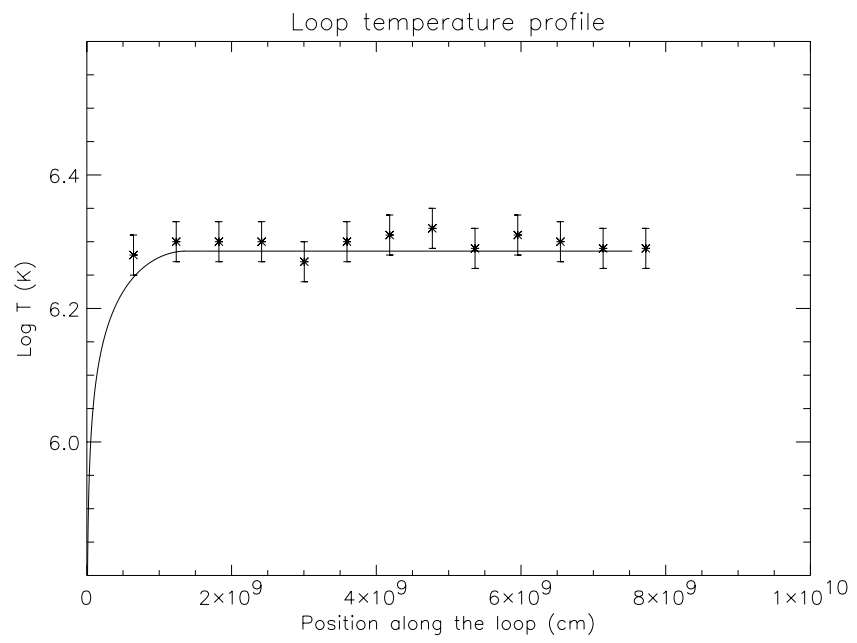

Fig. 14. Same as Fig. 8, for Case IV. An isothermal region has been added at the loop top to reproduce the coronal region of the loop. The theoretical model is assumed not to be thermally insulated at the base.

definition of the loop's right footpoint. However, the presence of a non-negligible conductive flux at the base of the model loop has three effects:

1. it steepens the temperature gradient at the loop base;

2. it shortens the length of the loop model;

3 . it changes its shape parameters.

The first of these effects points in the right direction for reproducing the nearly isothermal loops that are being observed by a number of authors, including the present work, since it confines the lower temperature portions of the loop to very short regions.

The second effect, on the contrary, makes the problem of total loop length worse, indicating that an even longer isothermal region is required in order to satisfy the observational constrains.

The third effect is important as the changes to the loop shape are very large. Unfortunately, the paucity of colder lines with non-negligible fluxes in our dataset, as well as the uncertainty in the definition of the loop right footpoint, from which a large fraction of their emission comes, prevent us from drawing any quantitative conclusion.

\subsection{Energy requirements of the loop model}

From the loop model it is possible to measure the total input energy required by the loop structures, given by the value of the radiative losses at the point $T_{\mathrm{M}}$.

From the data in Table 2 the power supply to the selected loop is $8.2 \times 10^{-4} \mathrm{erg} \mathrm{cm}^{-3} \mathrm{~s}^{-1}$. The uncertainties quoted in Table 2 for $p_{0}$ and $T_{\mathrm{M}}$ imply at least a factor 2 uncertainty for the evaluated input.

\subsection{Reliability of the results}

The present results depend on a number of theoretical quantities required to calculate the line emissivities. Uncertainties in these quantities may affect the results of any diagnostic technique making use of theoretical emissivities, as shown by Gianetti et al. (2000). Landi \& Landini (1999) discussed the effects of uncertainties in the radiative loss curves (adopted also in the present work) and concluded that the largest effects were due to element abundances and ion fractions. The effects of uncertainties in these two quantities are now discussed.

The crossing points of the curves displayed in Figs. 7, 9, 11 and 13 depend on element abundances, and any departure of the solar values from the adopted ones would be reflected in the crossing point. Since in the present work the crossing point is determined by low-FIP lines, with the only exception being $\mathrm{OV}$, the abscissa of the crossing point would not be changed by the choice of photospheric abundances, so that $T_{\mathrm{M}}$ would remain unchanged. However, since the photospheric abundances of low-FIP elements are around a factor 4 lower than the coronal ones, the $p_{0} S_{\mathrm{M}}$ value would be changed accordingly, so that the results of the comparison would be heavily changed. However, in the present dataset iron lines from Fex to Fe XVI are observed, and they provide a well-defined crossing point; the agreement of curves corresponding to lines emitted by other elements (especially $\mathrm{OV}$ ) with the $\mathrm{Fe}$ crossing point confirms that the adopted element abundances from Feldman (1992) are the same as the plasma values. Although the presence of a non-negligible conductive flux changes the shape of the loop, and therefore the curves of the cooler lines, these changes are not sufficient to alter the conclusions regarding the relative highFIP/low-FIP abundance.

Ion fractions also are a very important parameter to be considered, and changes in their values may have serious consequences for the results. The same study 
has therefore been repeated using the ion fractions from Arnaud \& Rotenflug (1985) for Fe also, and with Shull \& Van Steenberg (1982) and Mazzotta et al. (1998) ion fractions. In all cases the assumption of ionization equilibrium has been maintained. The results show some variability, but the overall behaviour is unchanged, the changes in the loop physical quantities are within the experimental uncertainties and the conclusions of the present work are not altered.

\subsection{Effects of the uncertainties in the loop selection}

As described in Sect. 3.2, the choice of the loop to be analyzed in the present work is made difficult mainly by the low spatial resolution of the CDS and EIT instruments. The selection and identification of the loop analyzed in the present work is reasonable, but some uncertainties are still left, especially in the definition of the right footpoint of the loop. This is probably the main weakness of the present work, and of all similar investigations based on observations from instruments with moderate spatial resolution like CDS.

This uncertainty has a large effect on the measurement of the colder line intensities, especially $\mathrm{Ov}$, whose emission is concentrated in the loop footpoints. For this reason, the measurement of the parameters governing the loop shape $\left(S_{\mathrm{M}} / S_{\mathrm{m}}\right.$ and $\left.n\right)$ is very uncertain. Therefore, the measured values reported in Table 2 should be treated with caution. The only result that can be drawn from the present study is that there is some evidence that the loop cross-section is not constant, since in this case the $\mathrm{OV}$ predicted line intensity would be far higher than observed, and also the intensity of the other colder lines would be largely overestimated.

The effects of the uncertainty in the loop selection on the other physical parameters of the loop are less important in the case where no isothermal region is added at the loop top, since the diagnostic technique relies mostly on the crossing point of the coronal lines. These are emitted mostly by the portions of the loop best defined in Fig. 5 .

On the contrary, the inclusion of the isothermal region forces us to exclude from the line intensity measurement a substantial part of the coronal section of the loop, so that the resulting line emission will be more sensitive to changes in the loop shape near the footpoints. This is particularly true in the non-thermally-insulated case (Case IV), where the length of the isothermal section is greatest.

Probably, the only unambiguous conclusion that can be drawn from the present analysis is that the standard model, as described in Paper I, fails in predicting the temperature profile of the loop, so that some substantial improvement must be introduced.

\subsection{Future work}

In the light of the results of the present work, the most important aspects of the loop model that can be investigated are:
- the use of a non-uniform heating function;
- the assumption of non-negligible energy flux at the loop footpoints.

Also, future work will include a generalization of the loop model, by releasing the constant pressure assumption and introducing the effect of flows in the model derived in Paper I.

Future detailed comparisons between the model and spectroscopic observations of active region loops must await the advent of spectrographs with much higher spatial and temporal resolution, so that the main ambiguities underlying the present analysis can be at least partially removed. In light of this, the future EUV Imaging Spectrograph (EIS) on board of the joint Japan/US/UK SOLAR-B satellite due to launch in 2005 , will provide a unique opportunity for detailed comparisons such as those described in the present work. This instrument will be designed to carry out high-resolution observations of large fields of view on the Sun at a much higher cadence than any previous spectrograph; its spatial resolution of $2^{\prime \prime}$ will be of great help in the determination of active region loop shapes.

\section{Conclusions}

In the present work CDS observations (part of JOP 54) of an active region loop are analyzed. CDS intensity maps and images from SOHO-EIT, SOHO-MDI and YohkohSXT, also available as part of JOP 54, have been used to determine the shape of the selected loop. The physical properties of the emitting plasma along the loop length have been determined via line ratio techniques; the filling factor of the selected loop has been measured and is found to be in the range $0.1-1$.

The diagnostic technique developed by Landini \& Landi (2002, Paper I) for the comparison of the loop model developed in Paper I and spectral observations has been applied to the selected active region loop. This diagnostic technique allows us to determine the parameters for the loop shape, the loop temperature profile, the loop length and the cross-section at its top. The results have been compared with the line ratio measurements of density and temperature and with the length and cross-section of the loop measured from intensity maps.

The comparison has shown that the standard model without an "ad-hoc" isothermal region at the loop top is not able to reproduce the observations, both when the loop model is assumed to be thermally insulated, and when this assumption is released. The presence of an isothermal region seems to indicate a better agreement between theory and observations in the case of a non-thermally-insulated 
loop. In case the loop is assumed to be thermally insulated, as in most of the loop models in the literature, no agreement is found.

However, the moderate spatial resolution of the instruments has left a margin of uncertainty in the selection of the active region loop shape; this uncertainty has important consequences for the analysis in the case where an isothermal portion of the loop is inserted in the analysis. Therefore, further comparisons using data from instruments with higher spatial (and temporal) resolution are required to provide more definitive results.

This work demonstrates the great potential of the loop diagnostic technique described in Paper I, points out some discrepancies between the theoretical model and observations that are larger than the uncertainties provided by the spatial resolution of the instruments, and indicates several starting points for future studies. It is highly recommended that the present analysis is repeated using imaging spectrographs with higher temporal and spatial resolution, such as the Extreme-Ultraviolet Imaging Spectrometer (EIS) on board Solar-B, due for launch in 2005.

Paper I argues that the presence of an isothermal region could be due to a number of different possible scenarios, like a non-uniform heating function, random heating and loop filamentation. Further work is in progress to extend the loop model and address this problem, in preparation for the Solar-B mission.

Acknowledgements. AB, IR and SKS would like to thank J. O. Stenflo and M. C. E. Huber for their encouragement and support. The work of AB and IR was partly supported by the Swiss National Science Foundation, grant No. 21-45083.95, and by a grant from the ETH-Zurich which is gratefully acknowledged. The work of ML and EL was partly supported by the Italian Space Agency (ASI) and the Research Ministry (MURST). The authors are also grateful to the CDS team, as well as the SOHO command staff whose help was invaluable for obtaining the observations. SOHO is a mission of International cooperation between ESA and NASA. Yohkoh is a mission of the Japanese Institute of Space and Astronautical Science.

\section{References}

Allen, R., Landi, E., Landini, M., \& Bromage, G. E. 2000, A\&A, 358, 332

Andretta, V., \& Jones, H. P. 1997, ApJ, 489, 375

Arnaud, M., \& Raymond, J. C. 1992, ApJ, 398, 394

Arnaud, M., \& Rothenflug, R. 1985, A\&AS, 60, 425

Aschwanden, M. J., Newmark, J. S., Delaboudinière, J. P., et al. 1999, ApJ, 515, 842

Aschwanden, M. J., Alexander, D., Hurlburt, N., et al. 2000a, ApJ, 531, 1129

Aschwanden, M. J., Nightingale, R. W., \& Alexander, D. 2000b, ApJ, 541, 1059

Binello, A. M., Landi, E., Mason, H. E., Storey, P. J., \& Brosius, J. W. 2001, A\&A, 370, 1071

Brekke, P., Kjeldseth-Moe, O., Brynildsen, N., et al. 1997a, Sol. Phys., 170, 163
Brekke, P., Kjeldseth-Moe, O., \& Harrison, R. A. 1997b, Sol. Phys., 175, 511

Brooks, D. H., Fischbacher, G. A., Fludra, A., et al. 1999, A\&A, 347, 277

Brosius, J. W., Davila, J. M., Thomas, R. J., \& Monsignori Fossi, B. C. 1996, ApJS, 106, 143

Brosius, J. W., Davila, J. M., \& Thomas, R. J. 1998, ApJ, 497, L113

Brosius, J. W., Davila, J. M., \& Thomas, R. J. 1999, ApJS, 119,255

Chae, J., Wang, H., Qiu, J., Goode, P. R., \& Wilhelm, K. 2000, ApJ, 533, 535

Delaboudinière, J.-P., Artzner, G. E., Brunaud, J., et al. 1995, Sol. Phys., 162, 291

Dere, K. P., Landi, E., Mason, H. E., Monsignori Fossi, B. C., \& Young, P. R. 1997, A\&AS, 125, 149

Dere, K. P., Landi, E., Young, P. R., \& Del Zanna, G. 2001, ApJS, 134, 331

Feldman, U. 1992, Phys. Scr., 46, 202

Feldman, U., Schühle, U., Widing, K. G., \& Laming, M. J. 1998, ApJ, 505, 999

Fludra, A., Brekke, P., Harrison, R. A., et al. 1997, Sol. Phys., 175,487

Gianetti, D., Landi, E., \& Landini, M. 2000, A\&A, 360, 1148

Handy, B. N., Acton, L. W., Kankelborg, C. C., et al. 1999, Sol. Phys., 187, 229

Harrison, R. A., Sawyer, E. C., Carter, M. K., et al. 1995, Sol. Phys., 162, 233

Judge, P. G., Woods, T. N., Brekke, P., \& Rottman, G. J. 1995, ApJ, 455, L85

Kankelborg, C. C., Walker, A. B. C. II, Hoover, R. B., \& Barbee, T. W. 1996, ApJ, 466, 529

Kankelborg, C. C., Walker, A. B. C. II, \& Hoover, R. B. 1997, ApJ, 491, 952

Kano, R., \& Tsuneta, S. 1995, ApJ, 454, 934

Kano, R., \& Tsuneta, S. 1996, PASJ, 48, 535

Kjeldseth-Moe, O., \& Brekke, P. 1998, Sol. Phys., 182, 73

Klimchuk, J. A., \& Porter, L. J. 1995, Nature, 377, 131

Landi, E., \& Landini, M. 1997, A\&A, 327, 1230

Landi, E., \& Landini, M. 1998, A\&A, 340, 265

Landi, E., \& Landini, M. 1999, A\&A, 347, 401

Landi, E., Landini, M., Dere, K. P., Young, P. R., \& Mason, H. E. 1999, A\&AS, 135, 339

Landi, E., Feldman, U., \& Dere, K. P. 2001a, ApJ, in press

Landi, E., Doron, R., Feldman, U., \& Doschek, G. A. 2001b, ApJ, 556, 912

Landini, M., Brković, A., Landi, E., Rüedi, I., \& Solanki, S. K. 1999, Space Sci. Rev., 87, 245

Landini, M., \& Landi, E. 2002, A\&A, 383, 653 (Paper I)

Lenz, D. D, De Luca, E. E., Golub, L., Rosner, R., \& Bookbinder, J. A. 1999, ApJ, 517, L155

Matthews, S. A., \& Harra-Murnion, L. K. 1997, Sol. Phys., 175,541

Mazzotta, P., Mazzitelli, G., Colafrancesco, S., \& Vittorio, N. 1998, A\&AS, 133, 403

Neupert, W. N., Newmark, J., Delaboudinière, J.-P., et al. 1998, Sol. Phys., 183, 305

Ogawara, Y., Takano, T., Kato, T., et al. 1991, Sol. Phys., 136, 1

Oluseyi, H. M., Walker, A. B. C. II, Santiago, D. I., Hoover, R. I., \& Barbee, T. W. II 1999, ApJ, 527, 992

Pauluhn, A., Rüedi, I., Solanki, S. K., et al. 1999, App. Opt., 38,7035 
Porter, L. J., \& Klimchuk, J. A. 1995, ApJ, 454, 499

Priest, E. R., Foley, C., Heyvaerts, J., et al. 1998, Nature, 393, 545

Priest, E. R., Foley, C., Heyvaerts, J., et al. 2000, ApJ, 539, 100

Reale, F., \& Peres, G. 2000, ApJ, 528, L45

Rosner, R., Tucker, W. H., \& Vaiana, G. S. 1978, ApJ, 220, 643

Scherrer, P. H., Bogart, R. S., Bush, R. I., et al. 1995, Sol. Phys., 162, 129
Shull, J. M., \& Van Steenberg, M. 1982, ApJSS, 48, 95

Spadaro, D., Lanzafame, A. C., Consoli, L., et al. 2000, A\&A, 359,716

The solar Maximum Mission Experiments 1980, Sol. Phys., 65,5

Tsuneta, S., Acton, L., Bruner, M., et al. 1991, Sol. Phys., 136, 37

Wilhelm, K., Curdt, W., Marsch, E., et al. 1995, Sol. Phys., 162,189

Young, P. R., Landi, E., \& Thomas, R. J. 1998, A\&A, 329, 291 